\title{
Association between Drinking Behavior and Activities of Daily Living in Community-dwelling Older Adults
}

\author{
Haewon Byeon ${ }^{1}$ and SungHyoun $\mathrm{Cho}^{2 *}$ \\ 1 Dept. of Speech Language Pathology \& Audiology, Nambu University, \\ Gwangju, South Korea, byeon@nambu.ac.kr \\ 2Department of Physical Therapy, Nambu University, Gwangju, South Korea, \\ geriatricpt1@naver.com
}

\begin{abstract}
As the elderly are more vulnerable to drinking than young adults, elucidation of the relationship between drinking habits of old age and $A D L$ is an important issue from the perspective of public health science. This study explores the relationship between drinking habits of the elderly and impaired Activities of Daily Living $(A D L)$ and provides basic materials to prevent impaired ADL in the old age. This study analyzed a total of 4,134 elderly people over the age of 65 who participated in 2011 Korean Longitudinal Survey of Aging. Drinking habits were classified into 'no drinking (no drinking experience in lifetime)', 'past drinking (have drinking experience in the past but presently do not drink)' and 'currently drinking'. ADL were classified into 'normal group' and 'Impaired $A D L$ group'. To compare the relationship between alcohol drinking and impaired ALD, the odds ratio $(O R)$ and $95 \%$ confidence intervals $(C I)$ were presented using hierarchical logistic regression. As the result of multivariate analysis (adjusted model), even after all confounding variables were adjusted, drinking had significant relationship with ADL impairment. Past drinkers has 6 times more risk of ADL impairment (OR=5.86, 95\% CI: 3.07-11.19) than nondrinkers and current drinkers 4 times more risk (OR=3.94, 95\% CI: 2.08-7.48) ( $p<0.05)$. As drinking in old age is associated with impaired ADL, moderation is required on drinkers for healthy aging.
\end{abstract}

Keywords: alcohol drinking, Activities of Daily Living, cognitive function, drinking habits

\section{Introduction}

Presently serious social problems are occurring in Korea such as rapid change in population structure due to extremely low birth rate and radical aging. In particular, aging is progressing with very high speed in Korea and the elderly population over the age of 65 already surpassed $7 \%$ of total population in 2000, entering aging society and as of 2014 , the elderly population reached $12.7 \%$, facing aged society in the near future [1]. It is predicted that aging will accelerate even faster and Korea will turn into a post-aged society with elderly population taking up $20 \%$ of total population in 2026 [1].

Recently, the goal of elderly health care is changing into 'healthy aging' which tries to extend life span and health [2]. For a successful aging, it is important to maintain activities of daily living (ADL) together with high cognitive capacity [901]. Especially, as impaired ADL is a state in which basic daily living activities previously possible becomes difficult due to diseases, which exerts negative effect on independent living, ADL is critical in the quality of life for the elderly.

* Corresponding author 
Numerous studies have found that impaired ADL has significant relationship with sociodemographic factors such as old age [3], low income [4] and low level of education [5], chronic diseases such as diabetes and cardio-cerebrovascular disease [6] and health behaviors such as drinking and smoking. Especially, it has been reported that drinking in old age not only has to do with reduced cognitive functions [7] but also is a risk factor for impaired ADL [8]. Many epidemiological studies have supported that excessive drinking is closely related to impaired ADL in old age [9] and recent systematic exploratory study [10] and Meta-research [11] scientifically verified that excessive drinking is a factor which has negative effect on cognitive health in old age.

Nevertheless, annual drinking rate of elderly population over the age of 65 in Korea is on the increase from $43 \%$ in 2007 to $46.8 \%$ in 2010 and high-risk drinking rate which means 7 glasses of Soju for male and 5 cups for female in one round of drinking also increased from $7.1 \%$ to $9.4 \%$ [1] for the same period. Besides, it was reported that the elderly who practice excessive drinking tend not just to consume larger quantity of alcohol in a single drinking round but to have higher frequency of drinking as well [1].

As the elderly are more vulnerable to drinking than young adults [12], elucidation of the relationship between drinking habits of old age and ADL is an important issue from the perspective of public health science. Nonetheless, studies on drinking habits have focused on specific regions $[13,14,15]$ or on specific classes such as the elderly in the rural areas [16] or the female elderly living alone [15] and there has been lack of studies on the relationship between the drinking of the elderly and ADL using representative data.

This study explores the relationship between drinking habits of the elderly and impaired ADL and provides basic materials to prevent impaired ADL in the old age.

\section{Methods}

\subsection{Subjects}

This study analyzed a total of 4,134 elderly people (1,754 males and 2,380 females) over the age of 65 who participated in 2011 Korean Longitudinal Survey of Aging (KLoSHA). KLoSHA is supervised by Korea Labor Institute and TNS Korea conducted the survey on commission from July 7, 2011 through December 2011 [1]. Sampling frame was (enumeration) districts of Population and Housing Census 2005 and 261,237 districts were set as sampling units. In 2011 survey, 10,000 people was set as maximum valid sample size and considering that average population over the age of 45 was 1.67 per household in 2000 Population and Housing Census, 1,000 sampling districts were selected. The method of the survey was computer-assisted personal interviews using laptop computers.

\subsection{Measurement}

Drinking habits were classified into 'no drinking (no drinking experience in lifetime)', 'past drinking (have drinking experience in the past but presently do not drink)' and 'currently drinking'. Using Korean Activities of Daily Living (K-ADL) [17], ADL was assessed as 'without help', 'with some help' or 'completely unable' on seven items (questions); change of clothes, washing face \& brushing teeth \& shampooing, bathing (shower), eating, moving around, using bathroom and toiletdistinction, which are minimal functions for independent living. After completion of the assessment, in order to classify the elderly into elderly group within normal range and dysfunctional elderly group, this study classified those who responded 'without help' for all items into normal-range group and those who answered 'with 
some help' or 'completely unable' to more than 1 item into impaired ADL group. The value of Cronbach's $\alpha$ which indicates internal consistency of ADL was 0.97.

Confounding variables included smoking (no smoking, past smoking, current smoking), gender, age (65 75, over 75), level of education (middle school and lower, over high school), level of income (gross income of the household), subjective health (good, fair, bad), diabetes (yes, no), high blood pressure (yes, no), stroke (yes, no), arthritis and rheumatism (yes, no), depression (yes, no), marital status (have spouse, divorced/separation, separation by death), cognitive function (normal, mild cognitive impairment, dementia), eyesight (good, fair, bad) and average monthly participation time in social activity (less than 1 hour, over 1 hour).

For level of cognitive function, Korean version of Mini-Mental Status Examination (K-MMSE) composed of 30 items was used [18]. The study classified the subjects with over 24 points as 'normal cognitive level', 20 to 23 points as 'mild cognitive impairment' and less than 19 points as 'dementia'.

For depression, CES-D10 [20] was used which standardized CES-D (the Center for Epidemiologic Studies Depression Scale) developed by Randolff [19] in Korean. CES-D10 is a depression screening test which reduced CES-D into 10 items and is in 4-point scale. Total score or point ranges from 10 to 40 and the higher the score, the more serious depression symptoms are, and the cutoff point was 24 . The value of Cronbach's $\alpha$ which represents internal consistency of CES-D10 was 0.87 .

\subsection{Statistical Analysis}

Chi-square test was used to compare smoking, gender, age, level of, level of, subjective health, diabetes, high blood pressure, stroke (yes, no), arthritis and rheumatism, depression, marital status, cognitive, eyesight and average monthly participation time in social activity of nondrinkers and drinkers. To compare the relationship between alcohol drinking and impaired ALD, the odds ratio (OR) and 95\% confidence intervals (CI) were presented using hierarchical logistic regression. IBM SPSS version 22.0 (IBM Inc., Chicago, Illinois) was used for all analyses.

\section{Results}

\subsection{General Characteristics of Population}

General characteristics of population are presented in Table 1. For age group, those over 65 years of age to less than 75 were $54.8 \%$ and over 75 were $45.2 \%$. Males were $42.4 \%$ while females were $57.6 \%$. For level of education, those with less than middle school education were $78.2 \%$ and the elderly with spouse were the majority with $66.3 \% .42 \%$ of the subjects perceived that their subjective health was poor and $27.9 \%$ responded that their eyesight is bad. For prevalence rate of diseases, diabetes was $21.7 \%$, high blood pressure $51.4 \%$, stroke $7.9 \%$, arthritis and rheumatism $34.1 \%$, depression $7.9 \%$, mild cognitive impairment $24.6 \%$ and dementia $21.6 \%$. Besides, $3.0 \%$ of the subjects responded that they participate in social activity for more than 1 hour per month. Current smokers were $12.3 \%$ while current drinkers were 57.4\%. Prevalence rate of impaired ADL was $8.0 \%$. 
Table 1.General Characteristics of Population

\begin{tabular}{|c|c|}
\hline Variables & $\mathrm{n}(\%)$ \\
\hline \multicolumn{2}{|l|}{ Impaired ADL } \\
\hline Yes & $330(8.0)$ \\
\hline No & $3,804(92.0)$ \\
\hline \multicolumn{2}{|l|}{ Sex } \\
\hline Male & $1,754(42.4)$ \\
\hline Female & $2,380(57.6)$ \\
\hline \multicolumn{2}{|l|}{ Age } \\
\hline $65-75$ & $2,265(54.8)$ \\
\hline $75-$ & $1,869(45.2)$ \\
\hline \multicolumn{2}{|l|}{ Level of education } \\
\hline Middle school and lower & $3,232(78.2)$ \\
\hline Over high school & $902(21.8)$ \\
\hline \multicolumn{2}{|l|}{ Level of income } \\
\hline Q1 & $1,598(38.7)$ \\
\hline Q2 & $1,118(27.0)$ \\
\hline Q3 & $932(22.5)$ \\
\hline Q4 & $486(11.8)$ \\
\hline \multicolumn{2}{|l|}{ Marital status } \\
\hline Have spouse & $2,741(66.3)$ \\
\hline Divorced/separation & $78(1.9)$ \\
\hline Separation by death & $1,315(31.8)$ \\
\hline \multicolumn{2}{|l|}{ Subjective health } \\
\hline Good & $680(16.4)$ \\
\hline Fair & $1,686(40.8)$ \\
\hline $\mathrm{Bad}$ & $1,768(42.8)$ \\
\hline \multicolumn{2}{|l|}{ Diabetes } \\
\hline Yes & $898(21.7)$ \\
\hline No & $3,236(78.3)$ \\
\hline \multicolumn{2}{|l|}{ High blood pressure } \\
\hline Yes & $2,125(51.4)$ \\
\hline No & $2,009(48.6)$ \\
\hline \multicolumn{2}{|l|}{ Stroke } \\
\hline Yes & $325(7.9)$ \\
\hline No & $3,809(92.1)$ \\
\hline \multicolumn{2}{|l|}{ Arthritis and rheumatism } \\
\hline Yes & $1,411(34.1)$ \\
\hline No & $2,723(65.9)$ \\
\hline \multicolumn{2}{|l|}{ Depression } \\
\hline Yes & $326(7.9)$ \\
\hline No & $3808(92.1)$ \\
\hline \multicolumn{2}{|l|}{ Cognitive function } \\
\hline Normal & $2,225(53.8)$ \\
\hline Mild cognitive impairment & $1,015(24.6)$ \\
\hline Dementia & $894(21.6)$ \\
\hline \multicolumn{2}{|l|}{ Eyesight } \\
\hline Good & $729(17.6)$ \\
\hline
\end{tabular}




$\begin{array}{ll}\text { Fair } & 2,235(54.1) \\ \text { Bad } & 1,155(27.9) \\ \text { Smoking } & \\ \text { No smoking } & 2,910(70.4) \\ \text { Past smoking } & 714(17.3) \\ \text { Current smoking } & 510(12.3) \\ \text { Drinking habits } & \\ \text { No drinking } & 1,054(25.5) \\ \text { Past drinking } & 708(17.1) \\ \text { Currently drinking } & 2,372(57.4) \\ \text { Average monthly participation time in social activity } & \\ \text { Less than 1 hour } & 4,012(97.0) \\ \text { Over 1 hour } & 122(3.0)\end{array}$

\subsection{Characteristics of Subjects with Impaired ADL}

Characteristics of subjects with impaired ADL are presented in Table 2. As the result of chi-squared test, there was significant difference between impaired ADL group and normal group in age, level of education, marital status, subjective health, diabetes, high blood pressure, stroke, arthritis \& rheumatism, depression, cognitive impairment, eyesight, smoking, drinking and average monthly participation time in social activity $(\mathrm{p}<0.05)$. The rate of impaired ADL was higher in those over the age of $75(13.4 \%)$, with high school education and lower $(8.9 \%)$, separated by death $(11.6 \%)$, with bad subjective health $(16.7 \%)$, diabetes $(10.8 \%)$, stroke $(29.5 \%)$, arthritis \& rheumatism (8.9\%), dementia $(27.0 \%)$, bad eyesight (16.1), past smoker $(11.5 \%)$, past drinker $(16.2 \%)$ and those with less than 1 hour of monthly social activities $(8.2 \%)(\mathrm{p}<0.05)$.

Table 2.Characteristics of Subjects with Impaired ADL, n (\%)

\begin{tabular}{llll}
\hline Variables & impaired ADL & \multirow{2}{*}{ P } \\
\cline { 2 - 3 } & No (n=3,804) & Yes (n=330) & 0.245 \\
\hline Sex & $1,624(92.6)$ & $130(7.4)$ & \\
Male & $2,180(91.6)$ & $200(8.4)$ & $<0.001$ \\
Female & & & \\
Age & $2,186(96.5)$ & $79(3.5)$ & $<0.001$ \\
65-75 & $1,618(86.6)$ & $251(13.4)$ & \\
$75-$ & & & \\
Level of education & $2,945(91.1)$ & $287(8.9)$ & \\
Middle school and lower & $859(95.2)$ & $43(4.8)$ & \\
Over high school & & & \\
Level of income & $1,462(91.5)$ & $136(8.5)$ & \\
Q1 & $1,044(93.4)$ & $74(6.6)$ & $<0.001$ \\
Q2 & $861(92.4)$ & $71(7.6)$ & \\
Q3 & $437(89.9)$ & $49(10.1)$ & \\
Q4 & & & \\
Marital status & $2,569(93.7)$ & $172(6.3)$ & \\
Have spouse & $59(92.2)$ & $5(7.8)$ & \\
Divorced/separation & $1,163(88.4)$ & $152(11.6)$ & \\
Separation by death & &
\end{tabular}


Subjective health

Good
Fair
Bad

$$
\begin{aligned}
& 675(99.3) \\
& 1,656(98.2) \\
& 1,473(83.3)
\end{aligned}
$$$$
5(0.7)
$$

Diabetes

Yes

No

High blood pressure

Yes

No

Stroke

Yes

No

Arthritis and rheumatism

Yes

No

Depression

Yes

No

Cognitive function

Normal

Mild cognitive impairment

Dementia

Eyesight

Good

Fair

Bad

Smoking

No smoking

Past smoking

Current smoking

Drinking habits

No drinking

Past drinking

Currently drinking

Average monthly participation time in social activity

Less than 1 hour

Over 1 hour
$801(89.2)$
$3,003(92.8)$
$1,938(91.2)$
$1,866(91.2)$
$229(70.5)$
$3,575(93.9)$

$1,285(91.1)$

$2,519(92.5)$

$3,559(93.5)$

245 (75.2)

2,193 (98.6)

958 (94.4)

653 (73.0)

$710(97.4)$

2,117 (94.7)

969 (83.9)

2,682 (92.2)

632 (88.5)

$490(96.1)$

$2,171(91.5)$

593 (83.8)

1,040 (98.7)
$30(1.8)$

295 (16.7)

$<0.001$

97 (10.8)

233 (7.2)

0.046

$187(8.8)$

$187(8.8)$

$<0.001$

$96(29.5)$

$234(6.1)$

$<0.001$

$126(8.9)$

204 (7.5)

$<0.001$

249 (6.5)

$81(24.8)$

$32(1.4)$

57 (5.6)

$241(27.0)$

$<0.001$

19 (2.6)

$118(5.3)$

$186(16.1)$

$<0.001$

$228(7.8)$

82 (11.5)

20 (3.9)

$<0.001$

$201(8.5)$

115 (16.2)

14 (1.3)

0.003

\subsection{The Relationship between Drinking Habits of Old Age and Impaired ADL}

The relationship between drinking habits of old age and impaired ADL is presented in Table 3. As the result of univariate analysis (crude model), drinking has significant relationship with ADL impairment. Past drinkers had 14 times more risk of ADL impairment ( $\mathrm{OR}=14.41,95 \% \mathrm{CI}$ : 8.20-25.32) than nondrinkers while current drinkers had 7 times more risk $(\mathrm{OR}=6.88$, 95\% CI: 3.98-11.88) $(\mathrm{p}<0.05)$.

As the result of multivariate analysis (adjusted model), even after all confounding variables were adjusted, drinking had significant relationship with ADL impairment. Past drinkers has 6 times more risk of ADL impairment (OR=5.86, 95\% CI: 3.07 - 
11.19) than nondrinkers and current drinkers 4 times more risk ( $\mathrm{OR}=3.94,95 \% \mathrm{CI}$ : $2.08-7.48)(\mathrm{p}<0.05)$.

Table 3.The Relationship between Drinking Habits of Old Age and Impaired ADL: Odds Ratio and $95 \% \mathrm{Cl}$

\begin{tabular}{lll}
\hline Drinking habits & Crude OR $(95 \%$ CI $)$ & Adjusted OR $(95 \%$ CI $)$ \\
\hline No drinking & 1.00 & 1.00 \\
Past drinking & $14.41(8.20,25.32)$ & $5.86(3.07,11.19)^{*}$ \\
Currently drinking & $6.88(3.98,11.88)$ & $3.94(2.08,7.48)^{*}$ \\
\hline
\end{tabular}

$* \mathrm{p}<0.05$

Confounding variables included smoking (no smoking, past smoking, current smoking), gender, age (65 75, over 75), level of education (middle school and lower, over high school), level of income (gross income of the household), subjective health (good, fair, bad), diabetes (yes, no), high blood pressure (yes, no), stroke (yes, no), arthritis and rheumatism (yes, no), depression (yes, no), marital status (have spouse, divorced/separation, separation by death), cognitive function (normal, mild cognitive impairment, dementia), eyesight (good, fair, bad) and average monthly participation time in social activity (less than 1 hour, over 1 hour).

\section{Discussion}

Since old age is the time when physical functions decline, it is important to maintain them for healthy aging. This study explored the relationship between drinking habits of old age and impaired ADL using national data.

Prevalence rate of impaired ADL was $8.0 \%$ in this study and was higher in females and old age. This result corresponds with that of Korea Institute for Health and Social Affairs that prevalence rate of impaired ADL was the highest among the female elderly and in old age over 75 [21]. In particular, since it has been reported that the elderly with impaired ADL have more chronic diseases, extra attention should be paid to the high-risk group for impaired ADL. In addition, as the elderly with problems of ADL require the help of caregivers and ultimately cause depletion of medical resources with the increase in the use of the medical system and increased burden of social security with the limitation of family support, it requires national-level support and attention.

In this study, drinking had significant relationship with impaired ADL. Even after adjusting all confounding variables, past drinkers had 6 times more risk and current drinkers had 4 times more risk of ADL impairment than nondrinkers. Numerous studies have reported that excessive drinking is a risk factor for ADL [22, 23]. In a follow-up study on 1,486 mid-aged Finnish for 22.8 years, excessive drinkers had around two times significantly more risk of cognitive impairment than nondrinkers [24]. In addition, 2-year follow-up study on 3,012 Chinese elderly over the age of 60 regarding the relationship between drinking habits and cognitive impairment found out that excessive drinkers who drank over 2 glasses of alcohol a day had 1.5 times more risk of cognitive impairment than nondrinkers [25]. This trend is similar in the progress of dementia as well; in the study by Xu et al. [26] on the elderly over the age of 60 , excessive drinkers had significantly higher onset of dementia than nondrinkers. Although it is difficult to directly compare the result of this study with those of preceding studies as they mainly focused on cognitive impairment, it can be inferred that drinking may have negative effect on ADL in that chronic drinking causes neurological damages.

The imitations of this study are as follows; first, as this study is a crosssectional one, drawn-outresults cannot be interpreted in causal relationships. To verify causal relationship, longitudinal studies are required in the future. Second, this study did 
not investigate period, amount and frequency of drinking, which requires careful interpretation of the results.

\section{Conclusion}

As drinking in old age is associated with impaired ADL, moderation is required on drinkers for healthy aging.

\section{References}

[1] Korea Labor Institute, Korean Longitudinal Survey of Ageing 2011, Sejong, (2014).

[2] S. M. Albert and V. A. Freedman, "Public health and aging: Maximizing function and well-being", Springer, New York, (2010).

[3] P. Baltes, and J. Smith, "New frontiers in the future of aging: Fromsuccessful aging of the younger old to the dilemmas of the fourth age", Gerontology, vol.49, (2003), pp.123-135.

[4] P. Lantz, J. House, R. Mero and D. Williams, "Stress, life events, andsocioeconomic disparities in health: results from the americans'changing livesstudy", Journal of Health and Social Behavior, vol.46, (2005), pp.274-288.

[5] H. Chiu, Y. Hsieh, L. Mau and M. Lee, "Associations betweensocioeconomic status measures and functional change among older people inTaiwan”, Ageing and Society, vol.25, (2005), pp.377-395.

[6] J. Katz, "Preferences, quality and the under-utilization of total jointarthroplasty", Medical Care, vol.39, (2001), pp.203-205.

[7] H. Byeon, Y. Lee, S. Lee, K. Lee, S. Moon, H. Kim, C. Hong, S. Son and S. Choi, "Association of alcohol drinking with verbal and visuospatial memory impairment in older adults: Clinical Research Center for Dementia of South Korea (CREDOS) study", IntPsychogeriatr, vol.27, no.3, (2015), pp.455461.

[8] J. Kim, S. Lee and S. Lee, "The relationship between health behaviors, health status, activities of daily living and health-related quality of life in the elderly", Journal of the Korean Gerontological Society, vol.30, no.2, (2010), pp.471-484.

[9] G. J. McDougall Jr, H. Becker, C. L. Delville, P. W. Vaughan and T. W. Acee, "Alcohol use and older adults: A little goes a long way:”, Int J Disabil Hum Dev, vol.6, (2007), pp.431-440.

[10] T. Etgen, D. Sander, H. Bickel and H. Förstl, "Mild cognitive impairment and dementia: the importance of modifiable risk factors", DtschArztebl Int. vol.108, (2011), pp.743-750.

[11] K. J. Anstey, H. A. Mack and N. Cherbuin, "Alcohol consumption as a risk factor for dementia and cognitive decline: meta-analysis of prospective studies", Am J Geriatr Psychiatry, vol.17, (2009), pp.542-555.

[12] J. W. Kim, D. Y. Lee, B. C. Lee, M. H. Jung, H. Kim, Y. S. Choi and I. G. Choi, "Alcohol and cognition in the elderly: a review", Psychiatry Investig, vol.9, (2012), pp.8-16,

[13] K. Min, J. Kim and C. Cha, "Comparison of the quality of life of the elderly inpatient with that of the normal elderly people", Korean J of Health EducationandPromotion, vol.17, no.2, (2000), pp.83-205.

[14] W. Jung and S. Choi, "Analysis of the factors involved in the elderly's quality of life", Journal of Dongnam Health College, vol.21, no.2, (2003), pp.193-205.

[15] S. Back, E. Ryu and K. Park, "A model construction for quality of life in single aged women", The Journal of Korean Academic Society of Adult Nursing, vol.20, no.2, (2008), pp.1-14.

[16] S. Son, "A Model construction of the quality of life in the rural elderly", Seoul National University, (2006).

[17] C. W. Won, K. Y. Yang, Y. G. Rho, S. Y. Kim, E. J. Lee, J. L. Yoon, K. H. Cho,H. Shin, B. Cho, J. Oh, D. Yoon, H. S. Lee and Y. S. Lee, "The development of Korean Activities of Daily Living (K-ADL) and Korean Instrumental Activities of Daily Living (K-IADL) scale", Journal of the Korean Geriatrics Society, vol.6, no.2, (2002), pp.107-120.

[18] Y. Kang, D. L. Na and S. Hahn, "A validity study on the Korean Mini-Mental State Examination (KMMSE) in dementia patients", Journal of the Korean Neurological Association, vol.15, no.2, (1997), pp.300-308.

[19] L. S. Randolff, "The CES-D scale: a self-report depression scale for research in the general population", Applied Psychological Measurement, vol.1, (1997), pp.385-401.

[20] J. Park and J. Jung, "The influence of depression on the life satisfaction of the elderly people living alone: A mediating effect of problem drinking”, Journal of the Korean Elderly Welfare, vol.47, (2010), pp.309-330.

[21] Korea Institute for Health and Social Affairs, "Elderly Living Conditions and welfare needs nationwide survey 2004",Korea Institute for Health and Social Affairs, Seoul, (2005).

[22] J. L. F. Santos, M. L. Lebrão, Y. A. O. Duarte, and F. D. D. Lima, "Functional performance of the elderly in instrumental activities of daily living: an analysis in the municipality of São Paulo", Brazil, Cadernos de SaúdePública, vol.24, no.4, (2008), pp.879-886. 
[23] F. Artaud, A. Dugravot, S. Sabia, A. Singh-Manoux, C. Tzourio and A. Elbaz, Unhealthy behaviours and disability in older adults: Three-City Dijon cohort study, vol.347, no.4240, (2013).

[24] J. J. Virta, T. Jarvenpaa, K. Heikkila, M. Perola, M. Koskenvuo, I. Raiha, J. O. Rinne and J. Kaprio, "Midlife alcohol consumption and later risk of cognitive impairment: a twin follow-up study", J Alzheimers Dis, vol.22, (2010), pp.939-948.

[25] J. Deng, D. H. Zhou, J. Li, Y. J. Wang, C. Gao and M. Chen, "A 2-year follow-up study of alcohol consumption and risk of dementia”, ClinNeurol Neurosurg, vol.108, (2006), pp.378-383.

[26] G. Xu, X. Liu, Q. Yin, W. Zhu, R. Zhang and X. Fan, "Alcohol consumption and transition of mild cognitive impairment to dementia", Psychiatry ClinNeurosci, vol.63, (2009), pp.43-49.
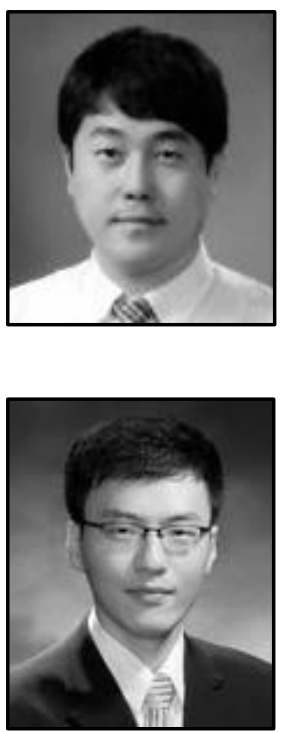

\section{Authors}

Haewon Byeon, he received the DrSc degree in Biomedical Science from Ajou University School of Medicine. He is a professor in Department of Speech Language Pathology \& Audiology and director of Speech Language Pathology Center inNambu University, Gwangju, Republic of Korea. His recent interests focus on mild cognitive impairment and motor speech disorders.

Sunghyoun Cho, he received the $\mathrm{PhD}$ degree in Physical Therapy from Daegu University. He is a professor in Department of Physical Therapy in Nambu University, Gwangju, Republic of Korea. His recent interests focus on Biomechanics and Therapeutic exercise. 
International Journal of Bio-Science and Bio-Technology

Vol.7, No.4 (2015) 